\title{
Primary malignant melanoma of the male urethra: a case report
}

\author{
Liqun Duan', Sanhe Liu', Yongzhi Li $^{3}$ \\ ${ }^{1}$ Department of Radiation Oncology, Hubei Cancer Hospital, Tongji Medical College, Huazhong University of Science and Technology, Wuhan, \\ China; ${ }^{2}$ Department of Urology, Hubei Cancer Hospital, Tongji Medical College, Huazhong University of Science and Technology, Wuhan, China; \\ ${ }^{3}$ Department of Urology, The Fourth Affiliated Hospital of China Medical University, Shenyang, China \\ Correspondence to: Yongzhi Li. Department of Urology, The Fourth Affiliated Hospital of China Medical University, Shenyang 110032, China. \\ Email: liyongzhi2324@hotmail.com.
}

\begin{abstract}
We present a rare case of a 69-year-old male patient with serendipitous urethral melanoma. He complained of dysuria and recurrent urinary retention and was initially diagnosed with benign prostatic hyperplasia. Accidentally, a dark-brown pigmented macula was found in the distal urethra at the end of transurethral prostatectomy when we exited the resectoscope, transurethral resection of the nidus and sent to pathological examination showed the characteristics of melanoma. No other lesions were found on further examination and the patient preferred a close follow-up cystoscopy rather than an immediate urethrectomy. Unsurprisingly, he relapsed in the urethra with the local disease three months later and we treated him with partial urethrectomy, followed by watchful waiting for 11 months. However, the patient was readmitted for hematuria, and ${ }^{18} \mathrm{~F}-\mathrm{FDG}$ PET-CT showed a large number of pelvic and bone metastatic lesions. Therefore, eight cycles of single-agent dacarbazine chemotherapy were administered, and the disease was demonstrated prolonged stabilization. Follow-up was conducted every 3 months, during which time palliative transurethral resection of the melanoma in the bladder was performed to control urinary tract infections. Although the prognosis of the disease is extremely poor, this patient has gained more than 50 months of overall survival and is alive to date.
\end{abstract}

Keywords: Case report; melanoma; male urethra; urethrectomy; dacarbazine

Submitted Jul 02, 2021. Accepted for publication Sep 24, 2021.

doi: $10.21037 /$ tcr-21-1150

View this article at: https://dx.doi.org/10.21037/tcr-21-1150

\section{Introduction}

Primary malignant melanoma of the genitourinary region is a rare disease; likewise, melanoma of the male urethra is rare. The neoplasm is highly invasive and associated with an extremely poor prognosis. The diagnosis is often delayed, and there is no standard treatment for urethral melanoma. Radical surgery is the initial choice of treatment followed by adjuvant chemotherapy or immunotherapy. We herein present a case of a 69-year-old man who was diagnosed with urethral melanoma by accident and underwent local resection, partial urethrectomy, dacarbazine chemotherapy, and palliative cystectomy after metastasis. He has survived for more than 50 months with definite efficacy to date. We present the following article in accordance with the CARE reporting checklist (available at https://dx.doi.org/10.21037/tcr-21-1150).

\section{Case presentation}

A 69-year-old man was admitted to our urology department in February 2017 with dysuria and recurrent urinary retention and was diagnosed with benign prostatic hyperplasia and bladder calculi. He had a history of hypertension and diabetes for half a year, and his blood pressure and blood glucose were well controlled. Transurethral resection of the prostate and cystolithotomy were performed. Accidentally, a 0.5 -cm dark-brown pigmented macula was found in the distal urethra at the end of the operation when the sheath was exited. Transurethral resection of the pigmented lesion revealed melanoma. His penile, scrotal and inguinal region examinations did not reveal any abnormalities. Given the high aggressiveness of the disease, an immediate urethrectomy was recommended. 

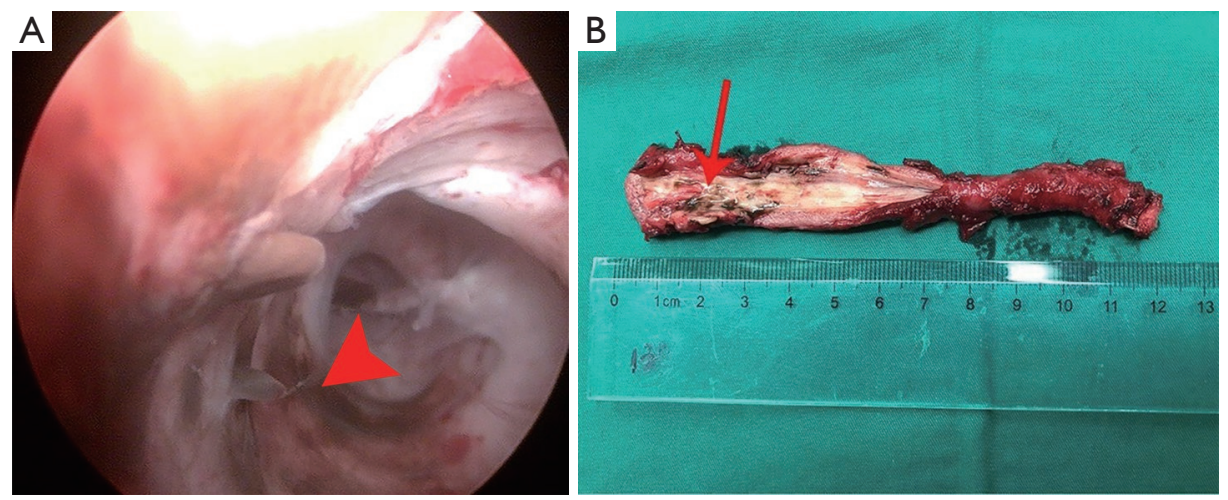

Figure 1 Cystourethroscopy and gross specimen photos presented brown lesions of the distal urethra mucosa. (A) Cystourethroscopy demonstrated a 0.5 -cm dark-brown pigment spot in the anterior urethra of the penis in June 2017. (B) Partial urethrectomy (the anterior urethra was removed $10 \mathrm{~cm}$ ) and the longitudinal opening revealed a small, pigmented lesion in the distal urethra.

However, he declined and preferred watchful waiting. In June 2017, cystourethroscopy demonstrated a $0.5-\mathrm{cm}$ dark-brown pigment spot in the pro-urethra of the penis, indicating a recurrence of the tumor (Figure 1A). A biopsy of the nidus was diagnosed as melanoma. Bilateral inguinal lymph node examination was negative, and computerized tomography examination of the chest, abdomen and pelvis showed no other lesions. Accordingly, partial urethrectomy and perineostomy without lymph node dissection were performed. Histopathological examination demonstrated a small group of tiny round heterotypic cells under the mucosa of the urethra, and pigment was seen in the plasma of some cells, which confirmed malignant melanoma with clean margins on both ends of the urethra (Figure 1B). Based on the early stages (pT1N0M0, stage A) of the tumor, no adjuvant therapy was performed. He was followed up every three months, and no recurrence was found eleven months postoperation. Then, he was readmitted to our department complaining of hematuria for approximately half a month in May 2018. ${ }^{18} \mathrm{~F}$-FDG PET-CT showed a large number of metastatic lesions. Multiple hypermetabolic lesions occurred in the bladder, the right side of the prostate and the posterior part of the prostate, the cavernous body of the urethra, the left $3 \mathrm{rd}$ anterior rib and 4th lumbar vertebra, and multiple thoracic vertebrae (Figure $2 \mathrm{~A}-2 \mathrm{H}$ ). Pelvic and bone metastasis of melanoma was diagnosed, and eight cycles of dacarbazine chemotherapy were adopted for the patient as an advanced maintenance therapy followed by a review every three months. The disease remained stable with a duration of urinary tract infection of one year. To address the lingering urinary tract infection, palliative transurethral resection of the melanoma in the bladder was performed in May 2019. The patient is currently alive and free from progression at 25 months after dacarbazine chemotherapy; his overall survival is currently more than 50 months. All procedures performed in studies involving human participants were in accordance with the ethical standards of the institutional and/or national research committee(s) and with the Helsinki Declaration (as revised in 2013). Written informed consent was obtained from the patient for publication of this case report and accompanying images. A copy of the written consent is available for review by the editorial office of this journal.

\section{Discussion}

Primary malignant melanomas of the genitourinary tract are rare, representing $<1 \%$ of all melanomas and accounting for $\sim 4 \%$ of urethral carcinomas (1). Primary urethral melanoma is three times more common in women than men (2) and tends to occur in older patients, averaging 65 years of age (3). Approximately 200 patients have been described in case reports or case series based on quick research of PubMed. The median survival is 28 months, with a 5 -year survival of approximately $10 \%$ (4). Symptoms of urethral melanoma are hematuria, urethrorrhagia, dysuria, strangury and palpable tumor. However, more cases have revealed atypical or asymptomatic symptoms (5). Our case was discovered only by accident at the end of a transurethral prostatectomy. Late diagnosis is common, as most patients start with irritating and obstructive voiding symptoms that can be misdiagnosed as a benign prostatic syndrome. In addition, $50 \%$ of patients exhibit metastatic disease at the time of diagnosis (6). 

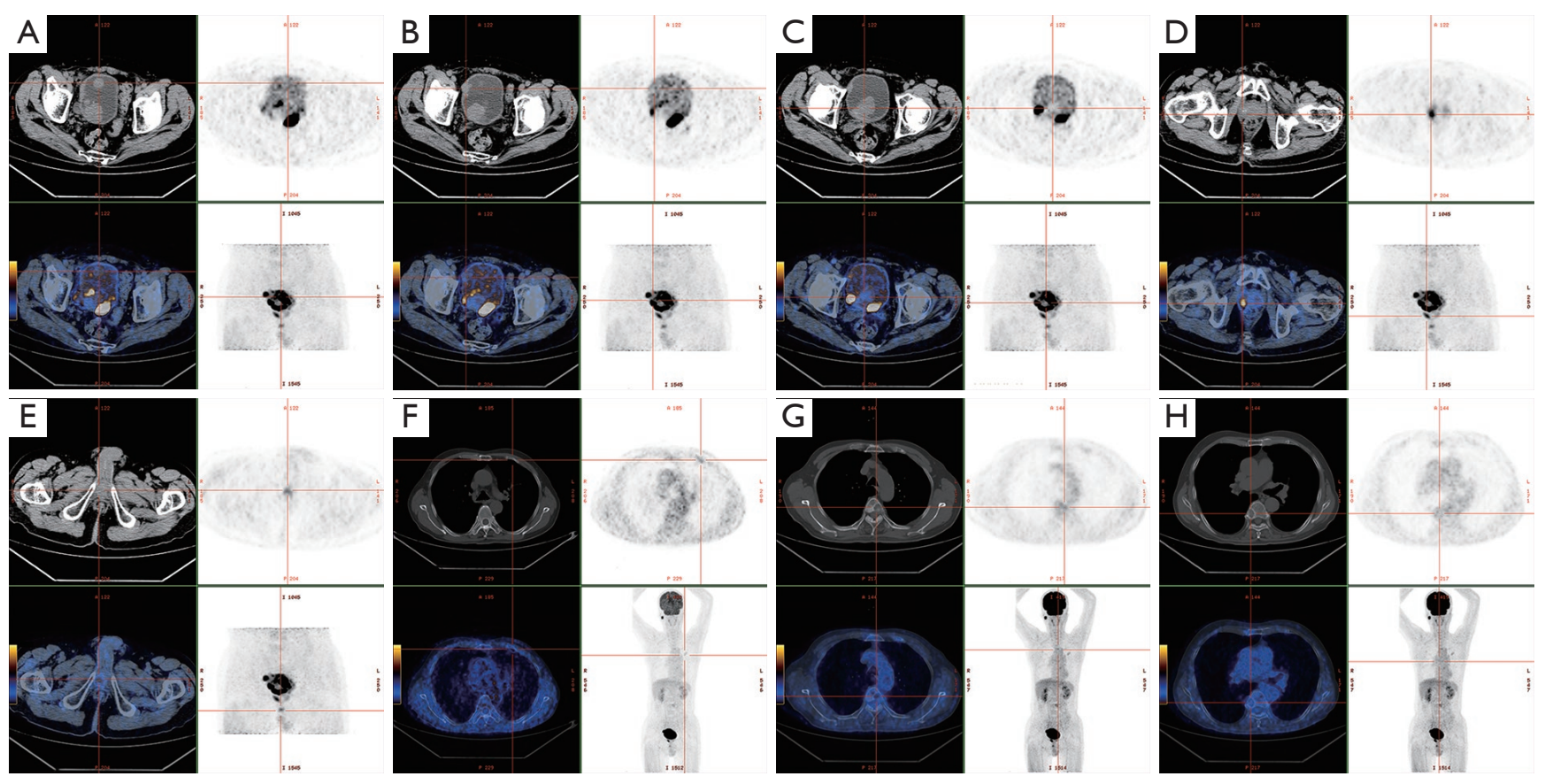

Figure $2{ }^{18}$ F-FDG PET-CT revealed multiple hypermetabolic lesions in the bladder (A-C), the right side of the prostate and the posterior part of the prostate (D), the cavernous body of the urethra (E), the left 3rd anterior rib (F) the 4th lumbar vertebra, and multiple thoracic vertebrae $(\mathrm{G}, \mathrm{H})$.

Malignant melanoma of the male genitourinary tract is most frequently localized on the urethra and penis, especially in the distal urethra. In humans, it is most frequently found in the fossa navicularis $(55 \%)$, urethra meatus $(5 \%)$, pendulous $(15 \%)$, bulbous $(10 \%)$, and prostatic urethra (15\%) (7). In our case, the lesion started from the distal urethra, gradually spread to the bladder, and finally metastasized to the distant body area.

Due to the highly aggressive characteristics of melanoma, early and definitive diagnosis is extremely important. Histopathological examination revealed neoplastic cells with vesicular nuclei in eccentric locations, conspicuous nucleoli, and atypical cells with eosinophilic cytoplasm aligned in niches (8) (Figure 3A,3B). Immunohistochemical markers, including S-100, HMB-45 and Melan A, aid in the differential diagnosis (Figure 3C-3E).

Sali et al. developed a common staging system as follows: stage A, tumor confined to the submucosa; stage B, tumor infiltrating the corpus spongiosum in men and the periurethral muscle in women; stage $\mathrm{C}$, tumor extending beyond the corpus spongiosum in men or periurethral invasion including the vagina, bladder, labia, or clitoris in women; and stage $\mathrm{D}$, tumor with metastasis to lymph nodes (9). Our patient initially had stage A disease, and one of the greatest controversies regarding this stage is the extent of surgery for localized disease. Some experts advocate total urethrectomy or total amputation of the penis and bilateral ilioinguinal node dissection (10). However, most scholars recommend that radical surgery carries no survival advantage for stage $C$ and stage $D$ patients $(11,12)$. Considering the rapid progression and poor prognosis of the tumor, expanding the scope of surgery would be accompanied by increased complications and decreased quality of life, so local excision with sufficient margins without bilateral complete inguinal lymphadenectomy was sufficient. Unfortunately, the patient exhibited metastasis to the bladder and bone. As the patient would not respond to the traditional six-course of dacarbazine chemotherapy because of his high metastasis tumor burden, we had extended two cycles of single-agent dacarbazine chemotherapy. Of course, this would have corresponded with image monitoring. After eight cycles of chemotherapy, the tumor in the bladder was nearly disappeared and the bone metastasizes were stable. The patient felt no symptoms with a well quality of life.

There is no standard treatment for urethral melanoma (13), 

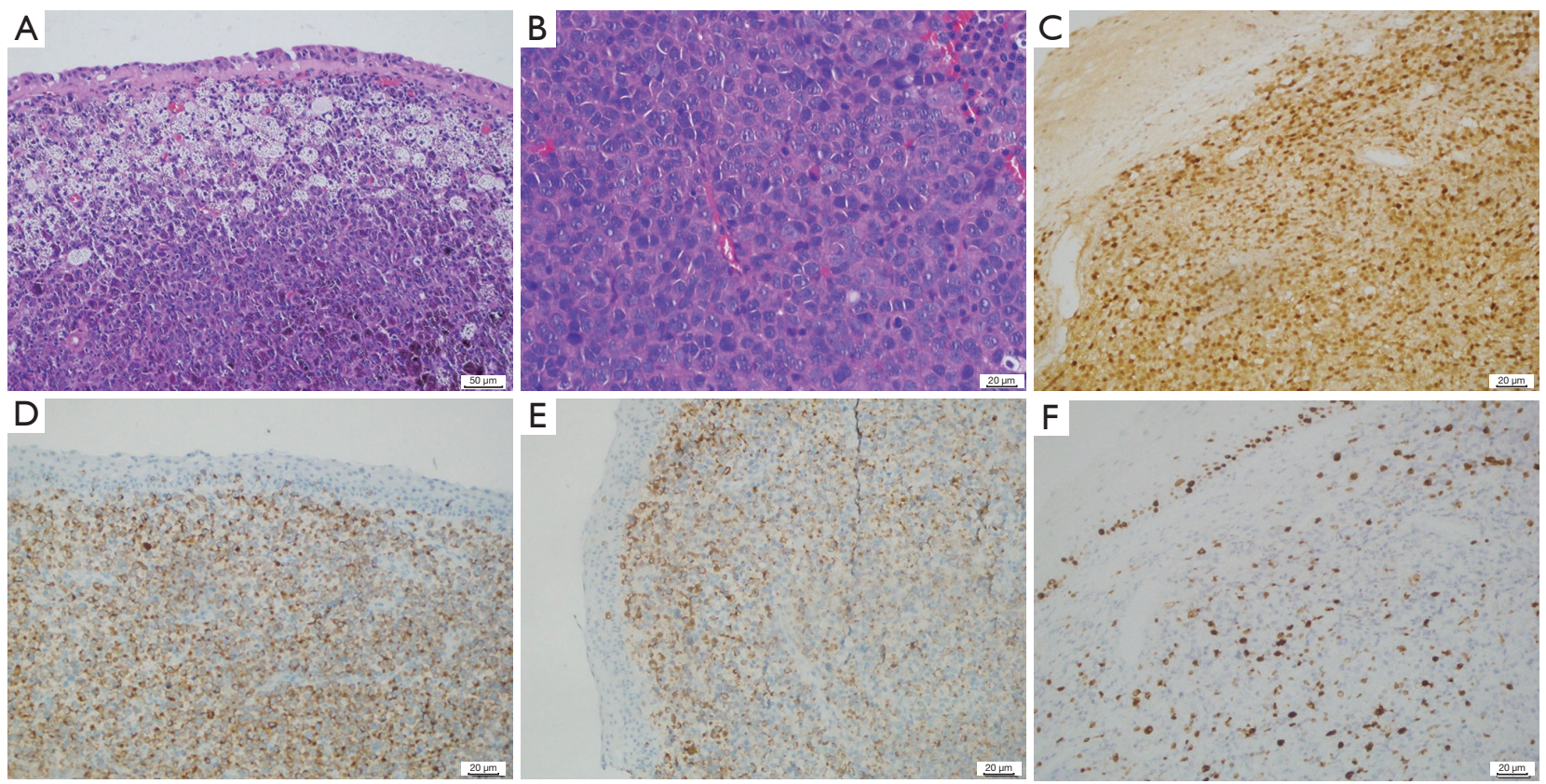

Figure 3 Histologic features of urethra melanoma. Histopathology revealed that the tumor was composed of sheets of epithelioid melanocytes, numerous atypical melanocytic cells with large hyperchromatic nuclei, and abundant cytoplasm (A, $\times 200$, scale bar: $50 \mu$ m; B, ×400, scale bar: $20 \mu \mathrm{m})$, staining method: hematoxylin and eosin staining. Positive S-100 (C, ×200, scale bar: $20 \mu \mathrm{m})$, Melan A (D, ×200, scale bar: $20 \mu \mathrm{m}), \mathrm{HMB}-45$ (E, ×200, scale bar: $20 \mu \mathrm{m})$, Ki-67 (Li: 40\%) (F, ×200, scale bar: $20 \mu \mathrm{m})$ immunohistochemical stain, respectively.

and clinical decision-making should be considered according to the presentation, clinical stage and general condition of the patient. Immune checkpoint blockade immunotherapy, including cytotoxic $\mathrm{T}$ lymphocyte-associated antigen (CTLA-4) and programmed cell death 1 (PD-1) receptor antibody treatment, has shown promising efficacy in the treatment of metastatic melanoma (14). However, mucosal melanoma has been reported to have a low response to immune checkpoint inhibitors (15). To the best of our knowledge, the patient might benefit from immunotherapy in the future. However, thus far, we have succeeded in treating the patient. We summarize the diagnosis and treatment experience as follows: (I) this patient had an occasional urethra melanoma, which means it was in the early stage of the disease when the patient was diagnosed. (II) Our immediate intervention and treatment, close monitoring and follow-up were of great benefit for the patient. (III) The dacarbazine chemotherapy then played an important role in the treatment of the patient. It is believed that our experiences will provide some guidance on the treatment of such cases.

\section{Acknowledgments}

Funding: This work was supported by the Cancer Research Program of the National Cancer Center (NCC201817B054).

\section{Footnote}

Reporting Checklist: The authors have completed the CARE reporting checklist. Available at https://dx.doi. org/10.21037/tcr-21-1150

Peer Review File: Available at https://dx.doi.org/10.21037/ tcr-21-1150

Conflicts of Interest: All authors have completed the ICMJE uniform disclosure form (available at https://dx.doi. org/10.21037/tcr-21-1150) and report that this work was supported by the Cancer Research Program of the National Cancer Center (NCC201817B054). The authors have no other conflicts of interest to declare. 
Ethical Statement: The authors are accountable for all aspects of the work in ensuring that questions related to the accuracy or integrity of any part of the work are appropriately investigated and resolved. All procedures performed in studies involving human participants were in accordance with the ethical standards of the institutional and/or national research committee(s) and with the Helsinki Declaration (as revised in 2013). Written informed consent was obtained from the patient for publication of this case report and accompanying images. A copy of the written consent is available for review by the editorial office of this journal.

Open Access Statement: This is an Open Access article distributed in accordance with the Creative Commons Attribution-NonCommercial-NoDerivs 4.0 International License (CC BY-NC-ND 4.0), which permits the noncommercial replication and distribution of the article with the strict proviso that no changes or edits are made and the original work is properly cited (including links to both the formal publication through the relevant DOI and the license). See: https://creativecommons.org/licenses/by-nc-nd/4.0/.

\section{References}

1. DeSimone RA, Hoda RS. Primary malignant melanoma of the urethra detected by urine cytology in a male patient. Diagn Cytopathol 2015;43:680-2.

2. Dogu GG, Yaren A, Tuncay L, et al. Primary malignant melanoma of the penis: a case report. Med Oncol 2012;29:1300-3.

3. Takamatsu D, Shiota $M$, Sugimoto M, et al. A case report of primary malignant melanoma of male urethra with distinct appearance in multiple regions. Int Cancer Conf J 2016;5:174-7.

4. Papeš D, Altarac S, Arslani N, et al. Melanoma of the glans penis and urethra. Urology 2014;83:6-11.

Cite this article as: Duan L, Liu S, Li Y. Primary malignant melanoma of the male urethra: a case report. Transl Cancer Res 2021;10(11):5005-5009. doi: 10.21037/tcr-21-1150
5. El-Safadi S, Estel R, Mayser P, et al. Primary malignant melanoma of the urethra: a systematic analysis of the current literature. Arch Gynecol Obstet 2014;289:935-43.

6. Fahmy O, Scharpf M, Fend F, et al. Feasibility of PenisPreserving Surgery for Urethral Melanoma: Proposal for a Therapeutic Algorithm. Clin Genitourin Cancer 2015;13:e411-3.

7. Zeighami S, Soltani M, Khajeh F, et al. Primary amelanotic melanoma of the male urethra: A rare entity and diagnostic challenge. Qatar Med J 2020;2020:11.

8. Li Y, Yuan H, Wang A, et al. Malignant melanoma of the penis and urethra: one case report. World J Surg Oncol 2014;12:340.

9. Sali GM, Nair KR, Bindhu MR, et al. Primary Malignant Melanoma of Urethra-Our Experience with 2 cases. Indian J Surg Oncol 2019;10:137-40.

10. Katz EE, Suzue K, Wille MA, et al. Primary malignant melanoma of the urethra. Urology 2005;65:389.

11. Baraziol R, Schiavon M, Fraccalanza E, et al. Melanoma in situ of penis: a very rare entity: A case report and review of the literature. Medicine (Baltimore) 2017;96:e7652.

12. Krishnan A, Caravaglio JV, Jhaveri F. Metastatic Malignant Melanoma of the Urinary Bladder in a Patient With Benign Prostatic Hyperplasia and Urethral Stricture. Clin Genitourin Cancer 2017;15:e119-21.

13. Hansen MF, Abel I, Clasen-Linde E. Primary malignant melanoma of the urethra in a patient with rheumatoid arthritis treated with methotrexate. BMJ Case Rep 2019;12:228033.

14. Tokita T, Kawahara T, Ito Y, et al. Primary amelanotic malignant melanoma of the male urethra with inguinal lymph node metastasis successfully controlled by nivolumab: A case report. Urol Case Rep 2018;18:54-6.

15. Watanabe K, Hasegawa G, Kashima K, et al. Primary malignant melanoma of the female urethra: A case report. Urol Case Rep 2021;34:101493. 